\title{
A Comparative Study of Genetic and Firefly Algorithms for Sensor Placement in Structural Health Monitoring
}

\author{
Guang-Dong Zhou, ${ }^{1}$ Ting-Hua Yi, ${ }^{2}$ Huan Zhang, ${ }^{1}$ and Hong-Nan $\mathrm{Li}^{2}$ \\ ${ }^{1}$ College of Civil and Transportation Engineering, Hohai University, Nanjing 210098, China \\ ${ }^{2}$ School of Civil Engineering, Dalian University of Technology, Dalian 116023, China \\ Correspondence should be addressed to Guang-Dong Zhou; zhougd@hhu.edu.cn
}

Received 11 August 2014; Accepted 1 October 2014

Academic Editor: Bo Chen

Copyright (c) 2015 Guang-Dong Zhou et al. This is an open access article distributed under the Creative Commons Attribution License, which permits unrestricted use, distribution, and reproduction in any medium, provided the original work is properly cited.

Optimal sensor placement (OSP) is an important task during the implementation of sophisticated structural health monitoring (SHM) systems for large-scale structures. In this paper, a comparative study between the genetic algorithm (GA) and the firefly algorithm (FA) in solving the OSP problem is conducted. To overcome the drawback related to the inapplicability of the FA in optimization problems with discrete variables, some improvements are proposed, including the one-dimensional binary coding system, the Hamming distance between any two fireflies, and the semioriented movement scheme; also, a simple discrete firefly algorithm (SDFA) is developed. The capabilities of the SDFA and the GA in finding the optimal sensor locations are evaluated using two disparate objective functions in a numerical example with a long-span benchmark cable-stayed bridge. The results show that the developed SDFA can find the optimal sensor configuration with high reliability. The comparative study indicates that the SDFA outperforms the GA in terms of algorithm complexity, computational efficiency, and result quality. The optimization mechanism of the FA has the potential to be extended to a wide range of optimization problems.

\section{Introduction}

The performance deterioration and the total collapse of largescale civil infrastructures induced by the environment and service loads highlight the importance of structural health monitoring (SHM) as a significant approach for the safe operation and the reasonable maintenance of structures. SHM, which involves an array of sensors to continuously monitor structural behavior, along with the extraction of damage-sensitive features from these measurements and the evaluation of current system health by analysis methods, can be used for rapid condition screening and aims to provide reliable information regarding the integrity of the structure in near real time [1-3]. At present, successful deployment and operation of long-term SHM systems on newly constructed structures and existing structures have been reported throughout the world [4-7]. In an SHM system, the sensor network provides original information indicating structural behavior for further parameter identification; therefore, the efficiency of an SHM system relies heavily on the reliability of the acquired data measured by the sensor networks on the structure. For the complexity of large-scale structures, such as long-span bridges and high-rise buildings, the degrees of freedom (DOFs) used to characterize structural performance count are on the order of thousands to tens of thousands. It is impossible to distribute sensors on all of the DOFs because of the high costs of data acquisition systems (sensors and their supporting instruments) and technology limitations $[8,9]$. Therefore, selecting optimal sensor placement (OSP) is a critical task before a sophisticated SHM system is designed and implemented on a real structure [10].

The problem of determining OSP has been investigated using a large number of interesting approaches and criteria in the past few decades, which can be seen from the abundance of literature. Among them, conventional gradient-based local optimization methods were unable to efficiently handle multiple local optima and may present difficulties in estimating the global minimum. They lack reliability in dealing with the OSP problem, because convergence to the global minimum is 
not guaranteed [11, 12]. Thus, the shift of OSP research away from classical deterministic optimization methods toward the use of combinatorial optimization methods based on biological and physical analogues has been motivated by the high computational efficiency and success rate of intelligent optimization methods. Many contributions regarding the adoption of intelligent optimization methods to the OSP problem have been recently made. The genetic algorithm (GA) based on the Darwinian principle of natural selection is a representative example and has proved to be a powerful tool for OSP. Yao et al. [13] demonstrated that the GA can replace the effective independence (EfI) method when using the determinant of the Fisher information matrix (FIM) as the objective function. Subsequently, a number of improvements have been employed to overcome drawbacks of the original GA. To accelerate convergence, the simulated annealing (SA) algorithm was integrated into the GA by Worden and Burrows [14] and Hwang and He [15] to extract the OSP in structural dynamic tests. To keep the sensor number constant during the genetic operation, the coding system was replaced by decimal two-dimensional array coding [16] or dual-structure coding [17]. With the purpose of improving the quality of solutions and convergence speed, two-quarter selection was adopted by Yi et al. $[18,19]$. The GA was also extended to the optimal wireless sensor placement, which has many constraints [20-22]. Particle swarm optimization (PSO), which is inspired by the movement of organisms in bird flocking or fish schooling, is another stochastic search technique and was successfully applied to the OSP problem $[23,24]$. Furthermore, the monkey algorithm (MA), which imitates the mountain-climbing process of monkeys, is considered to be an effective numerical method in solving complex multiparameter optimization problems. Several changes developed by Yi et al. made the MA excellent in terms of generating optimal solutions, as well as providing fast convergence in dealing with complicated OSP problems $[8,25,26]$.

Although the aforementioned methodologies demonstrated a strong capability, to some extent, in finding the acceptable solution for the OSP problem, the complex parameters and searching processes make those methods difficult to operate and susceptible to the application environment. The complexity of the optimal sensor configuration for largescale structures reveals the necessity for the development of efficient and robust algorithms to accurately explore the optimum solution. Recently, a new metaheuristic search algorithm, which is referred to as the firefly algorithm (FA), has been developed by Yang [27, 28]. The FA algorithm is based on the idealized behavior of the flashing characteristics of fireflies. A firefly tends to be attracted by other fireflies with high flash intensities. Previous studies indicate that the FA is particularly suited for parallel implementation and may outperform existing algorithms, such as PSO, GA, SA, and differential evolution, in terms of efficiency and success rates $[28,29]$. At present, the FA has been applied to a large number of optimization problems, including continuous, combinatorial, constrained, multiobjective, and dynamic optimization [30].
However, the coding system and the movement scheme in the FA make it suitable only for global numerical optimization problems with continuous variables. In this paper, some improvements, including the coding system, the suitable distance, and the movement scheme, are introduced, and a simple discrete firefly algorithm (SDFA) is proposed based on the FA such that the outstanding optimization mechanism of the FA can be applicable in the OSP problem with discrete variables. The remaining part of this paper is organized as follows: Section 2 presents a detailed description of the SDFA after an outline of the FA. Section 3 gives a brief introduction to the GA with the aim of facilitating performance comparison between the SDFA and the GA in the next numerical simulations. Section 4 shows the comprehensive evaluation of the SDFA for OSP with different criteria employing a longspan benchmark cable-stayed bridge. Finally, conclusions are drawn in Section 5.

\section{Firefly Algorithm}

2.1. Outline of Firefly Algorithm. The FA mimics the real firefly's swarm behaviors of communication, its search for food, and its process of finding mates. The optimization process of exploring the optimal solution is modeled in such a way that the firefly with low light intensity is attracted by the firefly with high light intensity and moves toward to it, such that the darker firefly has higher light intensity. Therefore, to establish the mathematical model of this movement, three hypotheses are adopted as follows: (1) the attractive action between two fireflies is only governed by the light intensity; (2) the light intensity of a firefly, which is deduced by the firefly's location, is proportional to the objective function; and (3) the light intensity decreases with increasing distance, such that the brighter firefly can only attract the fireflies within its attractiveness range. Then, the movement of firefly $i$ toward firefly $j$ is formulated as

$$
X_{i}^{t+1}=X_{i}^{t}+\beta_{0} e^{-\gamma r_{i j}^{2}}\left(X_{j}^{t}-X_{i}^{t}\right)+\alpha \varepsilon_{i}^{t},
$$

where $X_{i}$ and $X_{j}$ represent the locations of firefly $i$ and firefly $j$, respectively, the superscript $t$ denotes time, $\gamma$ means the light absorption coefficient, $r_{i j}$ is the distance between any two fireflies $i$ and $j$, and $\beta_{0}$ is the attractiveness at $r=$ 0 . The third item in (1) is a random vector, where $\alpha$ is a random parameter generated from the interval $[0,1]$ and $\varepsilon_{i}$ denotes a vector of random numbers drawn from a Gaussian distribution. Thus, the movement of firefly $i$ defined by (1) is not always directed to firefly $j$. More details can be found in references $[25,29,31,32]$.

The location of a firefly is simply coded using a spatial coordinate, which consists of real vectors and continuous variables. Subsequently, the distance between any two fireflies $i$ and $j$ is generally defined by the Euclidean distance $r_{i j}=$ $\left\|X_{i}-X_{j}\right\|_{2}$ or the $l_{2}$-norm. However, it is well known that, from the view of mathematics, the OSP is a specialized knapsack problem where some specified DOFs are selected to be placed by sensors, such that the structural performance can be described effectively. Thus, the parameters that are used for optimizing are states in which those DOFs are 
distributed by sensors and are discrete variables. As a result, the coding system and the movement strategies in the FA are inapplicable in the OSP problem with discrete variables. It is essential to do some modifications to the original FA, such that the underlying optimization concept of the FA can be moved to the OSP problem. Here, some improvements are integrated into the FA, and the SDFA is proposed to explore the optimal sensor configuration in structural health monitoring.

2.2. Simple Discrete Firefly Algorithm. Being originated from the FA, the SDFA is integrated by three parts: the coding system, the definition of distance between two fireflies, and the movement scheme. The coding system involves the code of each firefly in feasible space. The distance definition is responsible for describing the distance between two fireflies so that the movement can be realized. And the movement scheme gives the evolution rules of the SDFA. All of the three parts are introduced in next three sections.

2.2.1. Coding System. In the community of applying GA in finding the optimal sensor configuration, a widely used code approach is the one-dimensional binary coding system. Each individual in the population is coded by a one-dimensional binary string. In this code system, all of the candidate DOFs are put in a line. If the $s$ th DOF is placed by a sensor, the value of the sth element in the string is 1 . In contrast, if the $s$ th DOF is not placed by a sensor, the value of the sth element in the string is 0 . The total number of ones in the string is equal to the number of sensors that needs to be placed. This coding system is intuitive and easy to be initialized and operated. Here, in the SDFA, the one-dimensional binary coding system is employed. Each firefly in the population denotes a feasible sensor configuration, and the location of each firefly is represented by a one-dimensional binary string, as shown in Table 1. In the example of Table 1, it can be found that the 2nd, 3rd, 6th, and 9th DOFs are occupied by sensors. The total numbers of candidate DOFs and sensors are 10 and 4 , respectively, because the length of the string is 10 and the total number of ones is 4 in this firefly code. This coding method is very simple and intuitive, which is beneficial for the next optimization operation. When initializing the firefly population, the first $n$th elements in a string are set to 1 , and the left elements are set to 0 . Then, the shuffle algorithm is applied five times, such that the fireflies can be distributed in the feasible solution space uniformly as much as possible.

2.2.2. Distance between Two Fireflies. In the FA, the positions of the fireflies are defined in a Cartesian coordinate system, such that the distance between two fireflies can be easily calculated by the $l_{2}$-norm. However, in the SDFA, the positions of the fireflies are represented by binary strings. As a result, the $l_{2}$-norm is no longer suitable for indicating the distance between two fireflies. The Hamming distance [31], which counts the number of positions at which the corresponding symbols are different between two strings of equal length, has many similarities with the problem at hand and is adopted to indicate the distance between any two fireflies. Supposing
TABLE 1: An example of a firefly code.

\begin{tabular}{lllllllllll}
\hline DOF & 1 & 2 & 3 & 4 & 5 & 6 & 7 & 8 & 9 & 10 \\
\hline Code $\rightarrow$ & 0 & 1 & 1 & 0 & 0 & 1 & 0 & 0 & 1 & 0 \\
\hline
\end{tabular}

TABLE 2: Codes for firefly $i$ and firefly $j$.

\begin{tabular}{lllllllllll}
\hline Firefly $i \rightarrow$ & 1 & 0 & 1 & $\mathbf{0}$ & 0 & $\mathbf{1}$ & 0 & $\mathbf{1}$ & 0 & $\mathbf{0}$ \\
\hline Firefly $j \rightarrow$ & 1 & 0 & 1 & $\mathbf{1}$ & 0 & $\mathbf{0}$ & 0 & $\mathbf{0}$ & 0 & $\mathbf{1}$ \\
\hline
\end{tabular}

fireflies $i$ and $j$ with binary strings, the Hamming distance is equal to the number of ones in firefly $i$ XOR firefly $j$, which is

$$
r_{i j}=X_{i} \oplus X_{j}=\left(X_{i} \vee X_{j}\right) \wedge \neg\left(X_{i} \wedge X_{j}\right),
$$

where $X_{i}$ and $X_{j}$ are vectors, $\oplus$ means XOR, $\vee$ is logical disjunction, $\wedge$ represents logical conjunction, and $\neg$ denotes logical negation.

As a matter of fact, the Hamming distance represents the number of incongruous sensors between two sensor configurations and is equivalent to the number of elements in two strings whose values are different on the corresponding locations. Thus, the Hamming distance between firefly $i$ and firefly $j$ can be rewritten as

$$
r_{i j}=X_{i} \oplus X_{j}=\sum_{s=1}^{d}\left|x_{i, s}-x_{j, s}\right| .
$$

Generally, the number of sensors used for structural monitoring is predetermined, such that the total number of ones in any firefly is the same. If there is an incongruous sensor in firefly $i$, which is located at the sth DOF, it is not placed by a sensor in firefly $j$. There must be an incongruous sensor in firefly $j$, which is located at the $p$ th DOF, which is not placed by a sensor in firefly $i$. Then, the value of $r_{i j}$ is two. As a result, the distance $r_{i j}$, which is defined by (3), is always a nonnegative even number and is two times the number of incongruous sensors; this finding is beneficial for establishing the movement scheme. Therefore, the maximum value of $r_{i j}$ is $2 \omega$ (where $\omega$ is the total number of predetermined sensors), which implies that the sensors of firefly $i$ and the sensors of firefly $j$ are completely deployed on different DOFs in a structure. The minimum value of $r_{i j}$ is zero, which indicates that the sensors of firefly $i$ and the sensors of firefly $j$ occupy the same DOFs. Thus, the distance $r_{i j}$ has the range of [0 $\left.2 \omega\right]$. Table 2 gives an example of two codes for firefly $i$ and firefly $j$. The number of ones in firefly $i$ XOR firefly $j$ is four, and the number of incongruous sensors is two. Therefore, the corresponding Hamming distance between firefly $i$ and firefly $j$ is four.

2.2.3. Movement Scheme. The firefly movement in the Cartesian coordinate system is easily performed by changing the coordinate values, as formulated by (1). The distance of the movement is continuous and proportional to the attractiveness. However, the positions of the fireflies in the SDFA are defined by one-dimensional binary strings whose values are 0 and 1 . The firefly movement, which indicates that 
the values in the string are varied, can only be realized by changing 1 to 0 or 0 to 1 . On the other hand, the divergence of two fireflies originates from the incongruous sensors. Thus, replacing some incongruous sensors in a firefly may enhance its light intensity. Therefore, in the SDFA, the movement of firefly $i$ is operated by changing some elements in the string of firefly $i$ from values of 1 to 0 and changing some elements in the string of firefly $i$ from values of 0 to 1 , simultaneously. Additionally, to keep the number of ones constant, the time of changing 1 to 0 should be equal to the time of changing 0 to 1 . For this reason, the time of changing 1 to 0 or the time of changing 0 to 1 is defined as the movement distance. Under this definition, the nearest and farthest movement distance from firefly $i$ to firefly $j$ is 0 and $0.5 r_{i j}$, respectively. By introducing stochastic searching, the movement distance $d_{i j}$ from firefly $i$ to firefly $j$ is selected as

$$
d_{i j}=\operatorname{random}\left(1,0.5 r_{i j}\right) .
$$

Generally, the contribution of a sensor located on a DOF to the objective function cannot be predetermined. Therefore, it is difficult to judge which a sensor should be relocated. In the present paper, a semioriented movement scheme is proposed as follows.

Step 1. Calculate the difference between the strings of firefly $i$ and firefly $j$ :

$$
\Delta X_{j i}=X_{j}-X_{i}
$$

Step 2. Randomly select $d_{i j}$ elements from $\Delta X_{j i}$ with a value of 1 and change these elements to -1; again, randomly select $d_{i j}$ elements from $\Delta X_{j i}$ with a value of -1 and change these elements to 1 . The operated $\Delta X_{j i}$ is represented by $\left[\Delta X_{j i}\right]$.

Step 3. Replace the string of firefly $i$ by

$$
X_{i} \longleftarrow X_{i}+\left[\Delta X_{j i}\right] .
$$

In fact, the different light intensity between firefly $i$ and firefly $j$ comes from the $\Delta X_{j i}$ term, which is also induced by the incongruous sensors. The operated elements in the $\Delta X_{j i}$ term are randomly selected, because it is difficult to predict the influence of each element on the objective function. Therefore, replacing part of the $X_{i}$ by $\left[\Delta X_{j i}\right]$, which implies relocating some incongruous sensors, would enhance the light intensity of firefly $i$ with a high probability. However, the movement cannot guarantee that firefly $i$ moves in a desirable direction. Thus, the movement scheme is described as a semioriented movement scheme. These random factors in the movement scheme are also in accord with the random term in (1).

\section{Brief Description of Genetic Algorithm}

The GA is briefly described here to facilitate a comparison in next section. The GA, which was first proposed by Holland in 1975 [32], tries to imitate natural evolution by assigning a fitness value to each individual in the problem and by

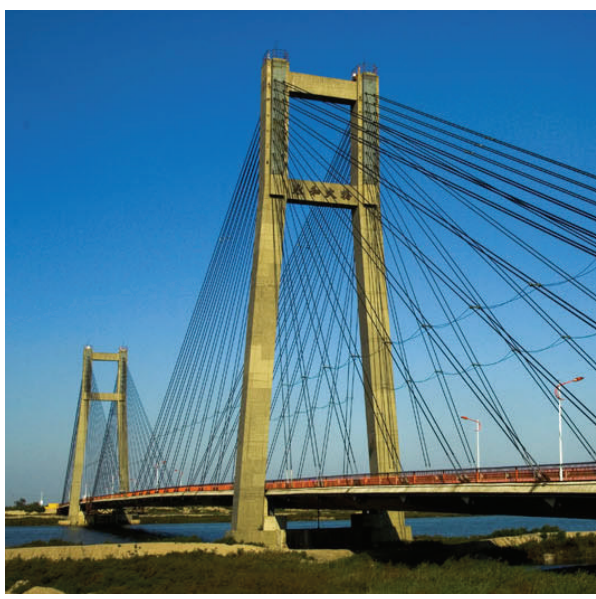

FIgURE 1: Overview of the cable-stayed bridge.

applying the principle of the survival of the fittest [33]. Each individual has a set of chromosomes that can be mutated and altered. Solutions can be represented by either onedimensional binary codes, dual-structure codes, or decimal codes. In this paper, the dual-structure coding method is employed to maintain a constant number of sensors. The evolution, which usually starts from a population of randomly generated individuals, is an iterative process and advances towards the next generation by applying genetic operators (crossover and mutation). An individual in the new population is generated by performing the crossover on two selected individuals from the current population and mutation on this generated individual [34]. The two individuals selected for crossover are chosen according to their fitness values. The individual with a good fitness value has a high probability of being chosen. The new generation of individuals is then used in the next iteration of the algorithm. Commonly, the iteration terminates when either a maximum number of generations have been produced or when a satisfactory fitness level has been reached for the population. The GA has a distinct advantage over traditional optimization techniques, which starts from a single point in the solution space. The details about the GA have been presented in references $[16,33,34]$.

\section{Numerical Example}

4.1. Bridge Description. The bridge employed for numerical simulation is a full-scale, cable-stayed bridge benchmark problem organized by the Center of Structural Monitoring and Control at the Harbin Institute of Technology [35], as shown in Figure 1. The bridge was opened to traffic in December 1987 and completely retrofitted in 2007. It comprises a main span of $260 \mathrm{~m}$ and two side spans of $25.15 \mathrm{~m}$ and $99.85 \mathrm{~m}$ each. The total length and width of the bridge are $519 \mathrm{~m}$ and $11 \mathrm{~m}$, respectively. The concrete bridge tower consists of two transverse beams with a height of $60.5 \mathrm{~m}$. 74 precast concrete girder segments are employed to integrate the main girder, and a total of 88 pairs of cables containing steel wires that are $5 \mathrm{~mm}$ in diameter are adopted to transfer the loads 


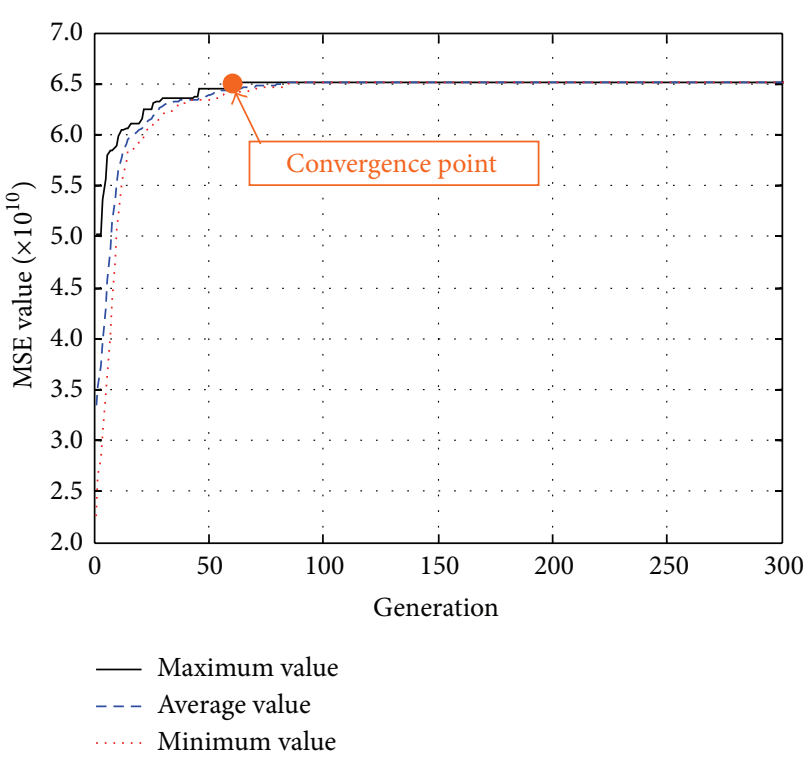

(a) SDFA

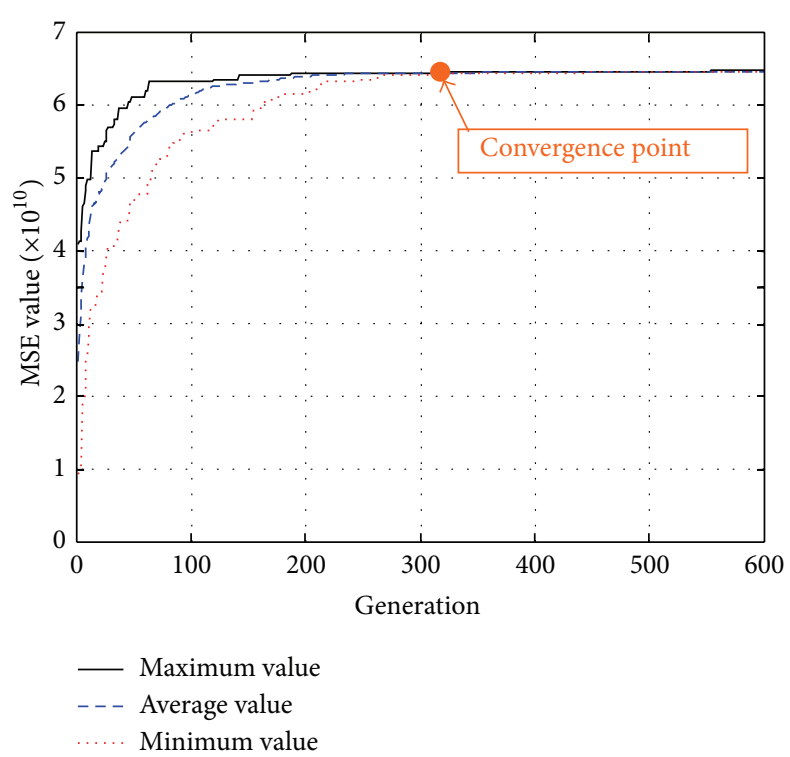

(b) GA

FIGURE 2: Iteration progress of the objective function.

from main girder to the two towers [36]. To understand the behavior of the bridge, an updated three-dimensional finite element model is also provided by the Center of Structural Monitoring and Control at the Harbin Institute of Technology [35]. The towers and the main girder were simulated by threedimensional beam elements, and the cables were simulated by linear elastic link elements. The concrete transverse beam at every $2.9 \mathrm{~m}$ was simplified by a mass element. The main girder was modeled as floating on the main tower, and all of the towers were fixed to the ground. The longitudinal restriction effect of the rubber supports was simulated by linear elastic spring elements. The model consists of 564 beam elements, 88 link elements, 160 mass elements, and 8 spring elements. Modal analysis has been conducted, and the results can be found in [35].

4.2. Results and Discussion. The proposed SDFA is evaluated by two frequently used, but quite different, objective functions. The first objective function is the modal strain energy (MSE). With this objective function, the OSP becomes a maximal optimization problem. The second objective function involves the modal assurance criterion (MAC), which induces the OSP to be a minimal optimization problem. More importantly, a comparative study is conducted between the SDFA and the GA, in terms of computational efficiency and result quality. To achieve this goal, the GA is also applied to find the optimal sensor configuration under the same conditions.

4.2.1. Optimization Based on Modal Strain Energy. Generally, it is desirable that most structural information is obtained through a set of sensors deployed on a structure, such that the structural behaviors can be described well. At present, the structural condition evaluation approaches based on the structural mode shapes and their derivations have been comprehensively explored. The MSE provides a rough measure of the dynamic contribution of each candidate sensor to the target mode shapes and implies that the DOFs capture most of the relevant dynamic features of the structure. The MSE helps to select those sensor positions with possible large amplitudes, which can increase the signal-to-noise ratio and improve the reliability of the mode identification results $[9,16]$. Therefore, the MSE is selected as the first objective function. Supposing that the mode shape matrix of a structure is $\Phi=\left[\phi_{1}, \phi_{2}, \ldots, \phi_{\omega}\right]$ ( $\omega$ is the number of mode shape vectors) and the number of measured points is $\omega$, the MSE can be expressed as

$$
H=\sum_{m=1}^{\omega} \sum_{n=1}^{\omega} \sum_{u \in \theta} \sum_{v \in \theta}\left|\phi_{u m} k_{u v} \phi_{v n}\right|
$$

where $\phi_{u m}$ is the $u$ th component in the corresponding $m$ th mode shape, $\phi_{v n}$ denotes the $v$ th component in the corresponding $n$th mode shape, $k_{u v}$ represents the stiffness coefficient between the $u$ th DOF and the $v$ th DOF, and $u \in \theta$ and $v \in \theta$ state that $u$ and $v$ are restricted to the locations where the sensors are placed.

Indeed, the improvements applied in the SDFA further simplify the FA algorithm, and only one parameter (i.e., the number of fireflies) needs to be preset. After being explored by a parametric study, the best value for firefly number is selected as 100, which allows the algorithm to achieve the best performance. Being different from the SDFA, the GA has several problematic parameters, such as the population size, the probabilities of selection, the crossover, and the mutation. Parametric studies are also conducted, and the appropriate values are determined. The simplicity and easy implementation of the SDFA is apparent. 
TABLE 3: Comparison of optimization results based on the MSE.

\begin{tabular}{lcccccrrr}
\hline $\begin{array}{l}\text { Sensor number } \\
\text { Methods }\end{array}$ & SDFA & GA & SDFA & GA & SDFA & GA & SDFA & GA \\
\hline $\begin{array}{l}\text { Maximum MAC off-diagonal value } \\
\quad\end{array}$ & & & & & & & & \\
$\quad$ Mean value $\left(\times 10^{10}\right)$ & 6.1416 & 6.1325 & 6.5271 & 6.5102 & 6.7232 & 6.7028 & 6.8195 & 6.8103 \\
$\quad$ Maximal value $\left(\times 10^{10}\right)$ & 6.2613 & 6.2537 & 6.5657 & 6.5325 & 6.7382 & 6.7215 & 6.8314 & 6.8217 \\
$\quad$ Standard deviation & 0.0291 & 0.0475 & 0.0280 & 0.4652 & 0.0165 & 0.3924 & 0.0121 & 0.2917 \\
\hline
\end{tabular}

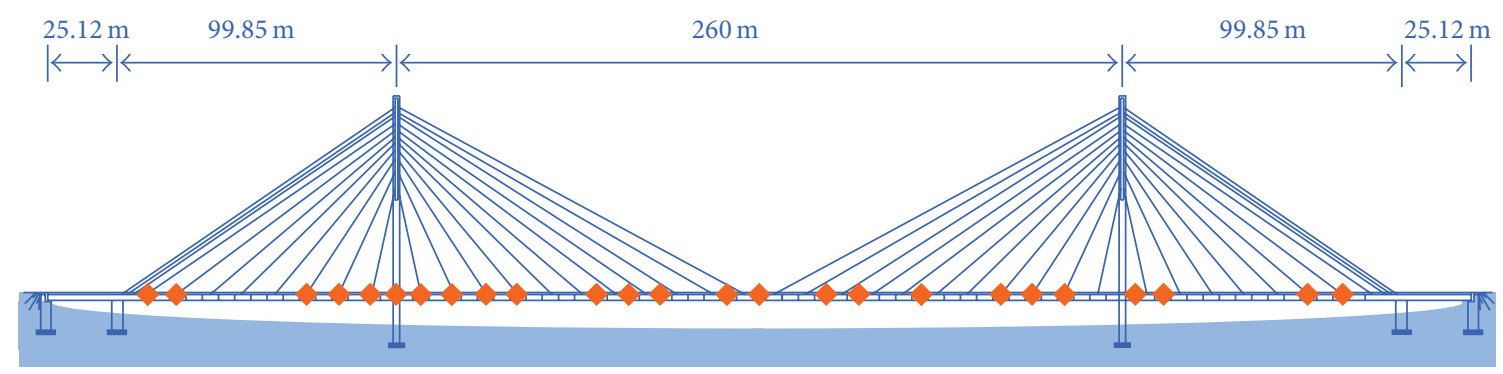

FIgURE 3: The optimal sensor configuration extracted by SDFA with the MSE.

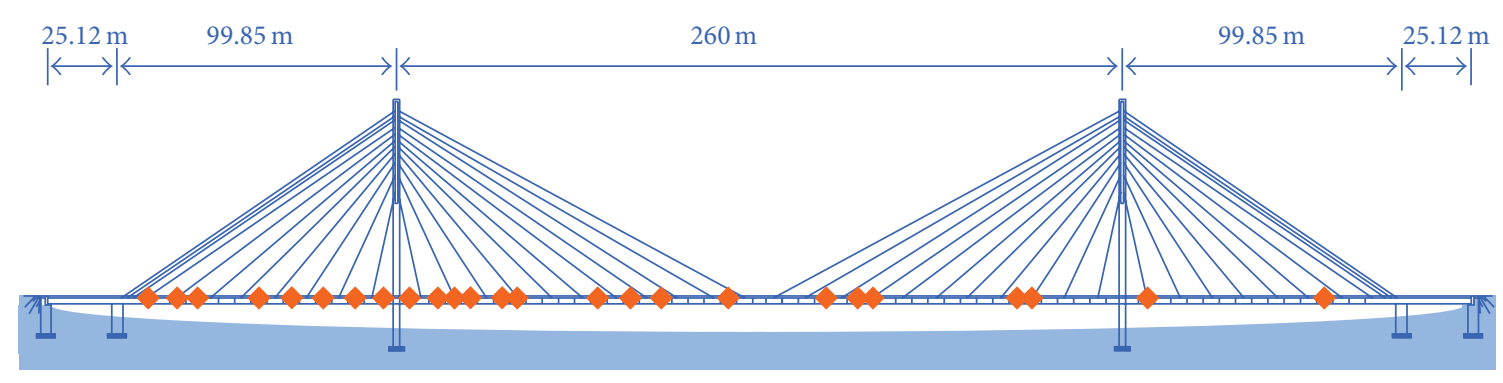

FIGURE 4: The optimal sensor configuration extracted by GA with the MSE.

Four scenarios with 20, 25, 30, and 35 sensors are simulated. In accordance with other heuristic optimization methods, the results extracted by the SDFA and the GA rely heavily on the randomly generated initial population. Therefore, to reduce the influence of the initialized individuals, the SDFA and the GA have been run 10 times with different stochastic initial populations in each occasion. The best iteration progress of the SDFA and the GA with 25 sensors are displayed in Figure 2, and the optimal locations of 25 sensors extracted by the SDFA and the GA with the aim of getting the maximal MSE value are illustrated in Figures 3 and 4, respectively. The statistical results of 10 runs for the four occasions are listed in Table 3. It can be seen from Figure 2 that both the SDFA and the GA can converge at the global optimum. In both Figures 2(a) and 2(b), the values of the objective function in the population increase with an increasing number of generations, and the average and minimum values of the objective function simultaneously approach the maximum value, which indicates good performance regarding optimum exploring. The effectiveness of the improvements adopted in the SDFA is validated. In the SDFA, the maximum MSE value tends to be a constant after 62 generations with a high speed. However, in the GA, converging to a constant spends 188 generations at an unacceptably low speed. Although increasing the number of individuals may reduce iteration generation, but the time of each generation becomes longer. As a result, the GA spends longer time finding the optimal solution than the SDFA. The high computational efficiency of the SDFA is revealed. Comparing Figures 3 and 4, it can be found that the optimal sensor locations extracted by the SDFA distribute on the span uniformly. However, the sensors in the optimal sensor configuration found by the GA crowd near the left tower, and the vibration and mode shapes of the right part of the bridge cannot be described clearly. Thus, the sensors in the optimal sensor configuration extracted by the SDFA are used with high efficiency and the identified mode shapes are more visual. The statistical results of each ten optimal solutions are listed in Table 3. In the table, the mean value and the maximal value represent the average and the maximal values of the ten optimal solutions, respectively, which indicate the superiority of the optimal solution; the standard deviation denotes 


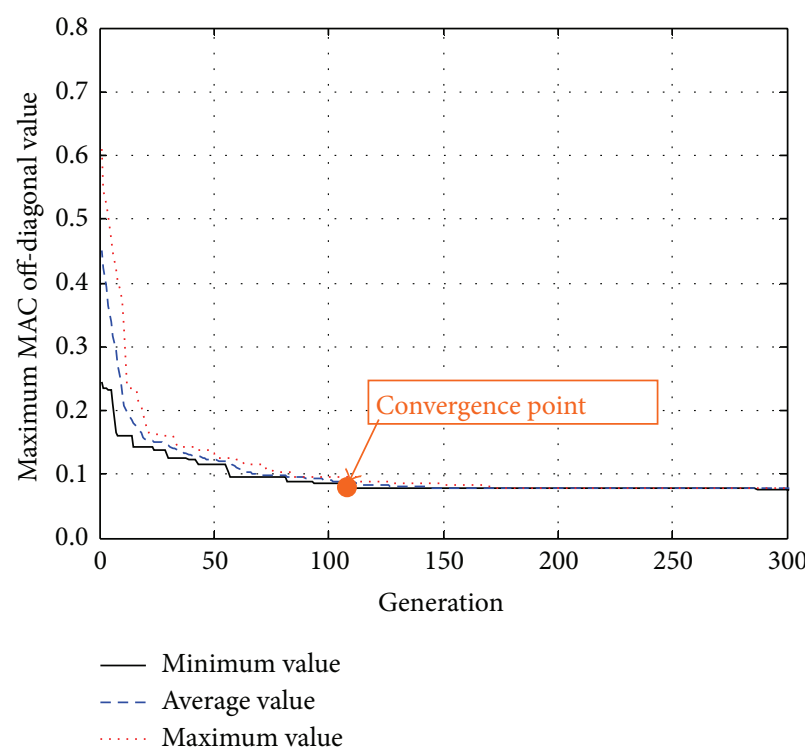

(a) SDFA

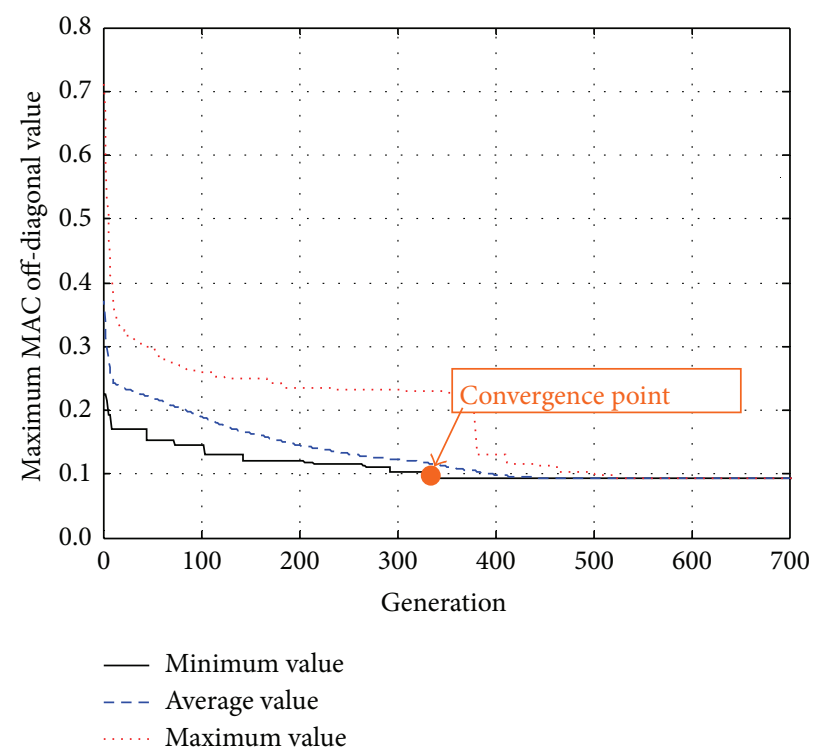

(b) GA

FIGURE 5: Iteration progress of the objective function.

the variability of the ten best solutions, which indicates the robustness of the algorithm. In every occasion, as listed in Table 3, the mean and maximum values of the MSE searched by the SDFA are larger than that explored by the GA. Thus, the solutions found by the SDFA have better quality than those extracted by the GA. Simultaneously, the standard deviation of the 10 results found by the SDFA in all four occasions are smaller than that searched by the GA, which shows that the SDFA can solve the OSP problem with high reliability and strong robustness.

4.2.2. Optimization Based on Modal Assurance Criterion. The vibration-based structural condition assessment methodologies require that the measured mode shapes are discriminable from each other, such that they can be reliably identified. The modal assurance criterion (MAC) proposed by Carne and Dohrmann [16] provides a simple metric to check the linear dependence of the mode shapes. A small maximum off-diagonal term of the MAC matrix implies less correlation between corresponding mode shape vectors and high distinguishability among the identified mode shapes. Thus, the MAC off-diagonal terms are adopted to evaluate the sensor configuration. The MAC is defined as

$$
\mathrm{MAC}_{m n}=\frac{\phi_{m}^{T} \phi_{n}}{\sqrt{\left(\phi_{m}^{T} \phi_{m}\right)\left(\phi_{n}^{T} \phi_{n}\right)}}
$$

where $\phi_{m}$ and $\phi_{n}$ represent the $m$ th and $n$th column vectors in matrix $\Phi$, respectively, and the superscript $T$ denotes the transpose of the vector. With this definition, the values of the MAC range from 0 to 1 , where 0 indicates that the modal vector is easily distinguishable and 1 indicates that the modal vector is fairly indistinguishable [19].
Consulting the numerical simulation performed in Section 4.2.1, the same parameters of the SDFA and the GA are adopted. The same four occasions are investigated, and each occasion is also calculated 10 times by the two methods. The best convergence process for the two optimization approaches are shown in Figure 5. The MAC values computed by the best sensor configurations obtained by the two methods are illustrated in Figure 6. To clearly show the MAC values of each mode, the maximum MAC off-diagonal values in each of the modes calculated by the SDFA and the GA are compared in Figure 7. The optimal sensor configurations found by the SDFA and the GA are displayed in Figures 8 and 9 , respectively. The statistical data of the 10 runs in the four occasions are also listed in Table 4 . The mean value, the minimal value, and the standard deviation have the similar meaning as that in Table 3 . From the convergence process in Figure 5(a) and the optimal results in Figure 6(a), the strong ability to search for the global optimal solution in the SDFA is again demonstrated from the minimal optimization problem. When comparing the iteration progress in Figure 5(a) with that in Figure 5(b), the higher computational efficiency of the SDFA is further validated. Investigating the optimization results illustrated in Figures 6, 7, 8, and 9, a more desirable sensor configuration can be acquired when using the SDFA. The statistical results listed in Table 4 also indicate that the SDFA has higher reliability and stronger robustness than the GA.

\section{Conclusions}

Because finding the optimal sensor locations under a certain evaluation criterion is a complicated nonlinear optimization problem, traditional optimization methods often encounter many insurmountable difficulties in solving this problem. 
TABLE 4: Comparison of optimization results based on the MAC.

\begin{tabular}{lcccccrrrr}
\hline $\begin{array}{l}\text { Sensor number } \\
\text { Methods }\end{array}$ & SDFA & GA & SDFA & GA & SDFA & GA & SDFA & GA \\
\hline $\begin{array}{l}\text { Maximum MAC off-diagonal value } \\
\quad\end{array}$ & & & & & & & & & \\
$\quad$ Mean value & 0.0826 & 0.0937 & 0.0806 & 0.0904 & 0.0793 & 0.0854 & 0.0755 & 0.0796 \\
$\quad$ Minimal value & 0.0659 & 0.0783 & 0.0614 & 0.0727 & 0.0587 & 0.0657 & 0.0561 & 0.0593 \\
$\quad$ Standard deviation & 0.0128 & 0.0203 & 0.0129 & 0.0212 & 0.0116 & 0.0195 & 0.0113 & 0.0221 \\
\hline
\end{tabular}

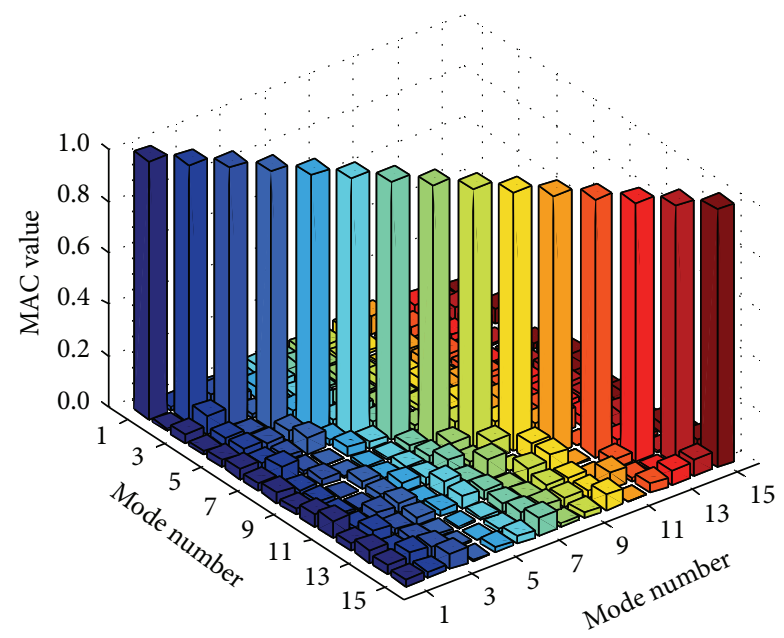

(a) SDFA

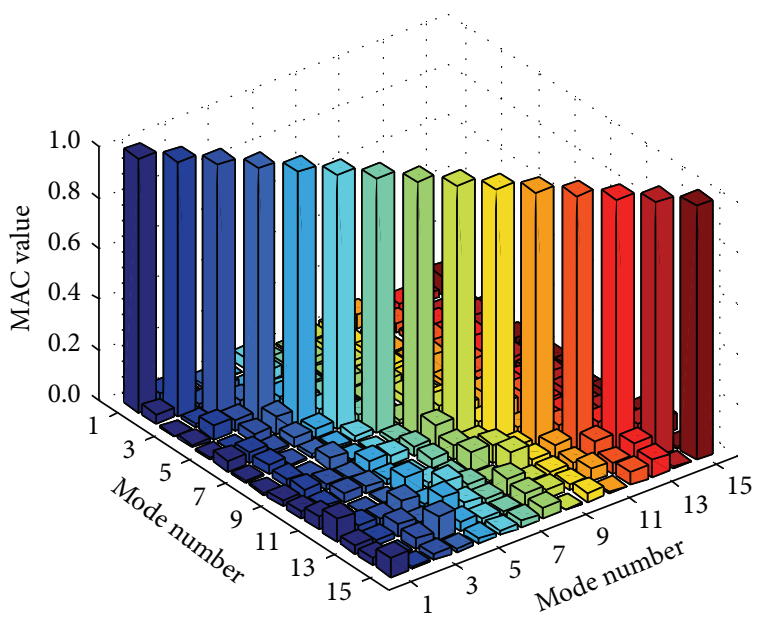

(b) GA

FIGURE 6: MAC values obtained from the optimal sensor configuration.

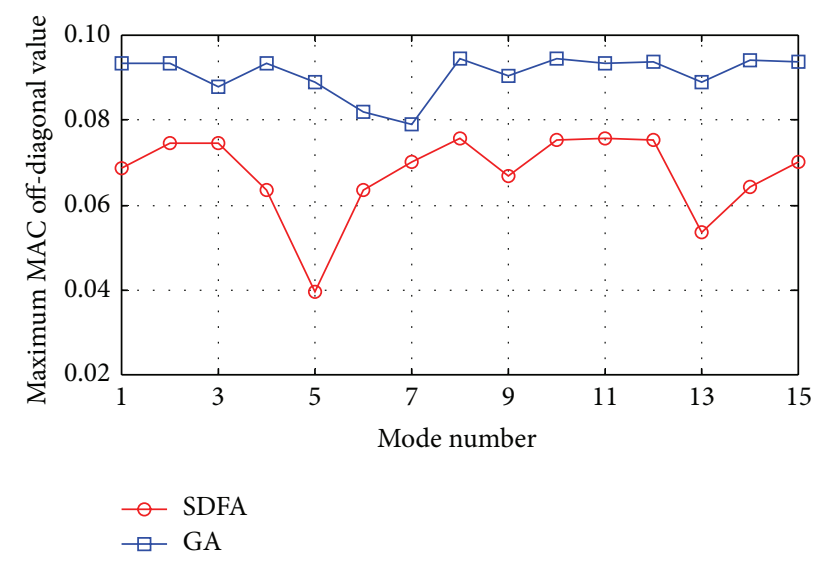

FIGURE 7: Maximum MAC off-diagonal values in each of the modes.

Intelligence optimization algorithms such as the GA and the FA provide powerful approaches to overcome these obstacles. Before implementing a comparative study between the GA and the FA regarding their performance in finding the optimal sensor configuration, some improvement are developed based on the basic FA and the SDFA is proposed. If all candidate DOFs can be accessible in real-word practice, the developed sensor placement method is applicable in any type of tethered sensor. The performance of the SDFA and the GA is compared using a numerical example with a long-span benchmark cable-stayed bridge. Some conclusions are drawn as follows.

(1) The one-dimensional binary coding system and the Hamming distance can rationally describe the status of fireflies in the feasible sensor configuration space. The semioriented movement scheme provides an effective tool to move the original movement defined in the Cartesian coordinate system to the onedimensional binary coding system. These improvements make the underlying optimization mechanism of the FA applicable in discrete optimization problems.

(2) In the case study, the improved SDFA shows good performance, both in the maximal value problem and the minimal value problem. The simulation results indicate that the SDFA has smooth convergence progress. The effectiveness of the proposed improvements is validated, and the strong capability of FA in finding the global optimization is also revealed.

(3) Compared with the widely accepted GA, the SDFA, which has only one problematic parameter, can be implemented more easily. Regarding both the MSE and MAC criteria, the SDFA shows superior computational efficiency and robustness versus the GA. The optimal solution extracted by the SDFA is also more desirable than that provided by the GA. 


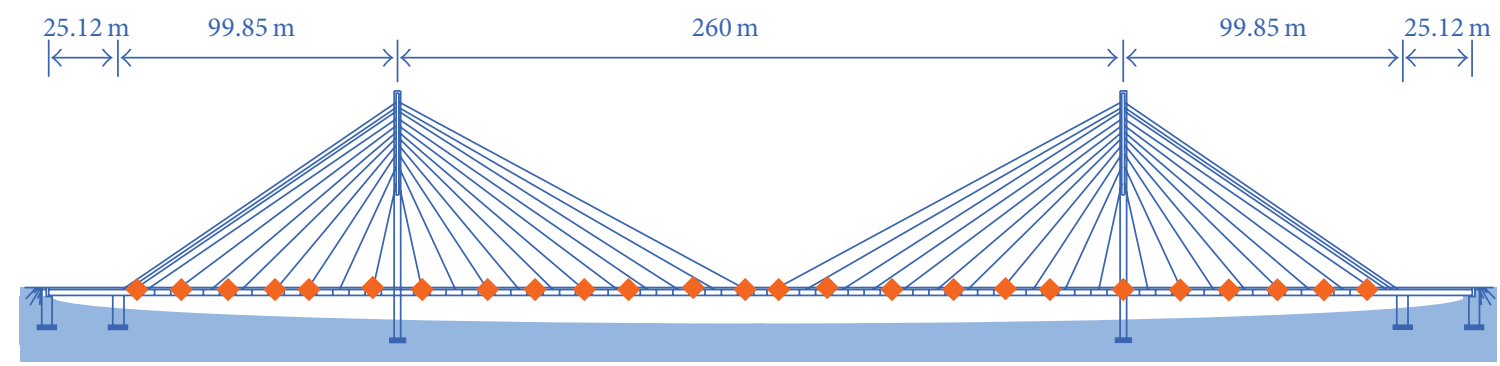

FIGURE 8: The optimal sensor configuration extracted by SDFA with the MAC.

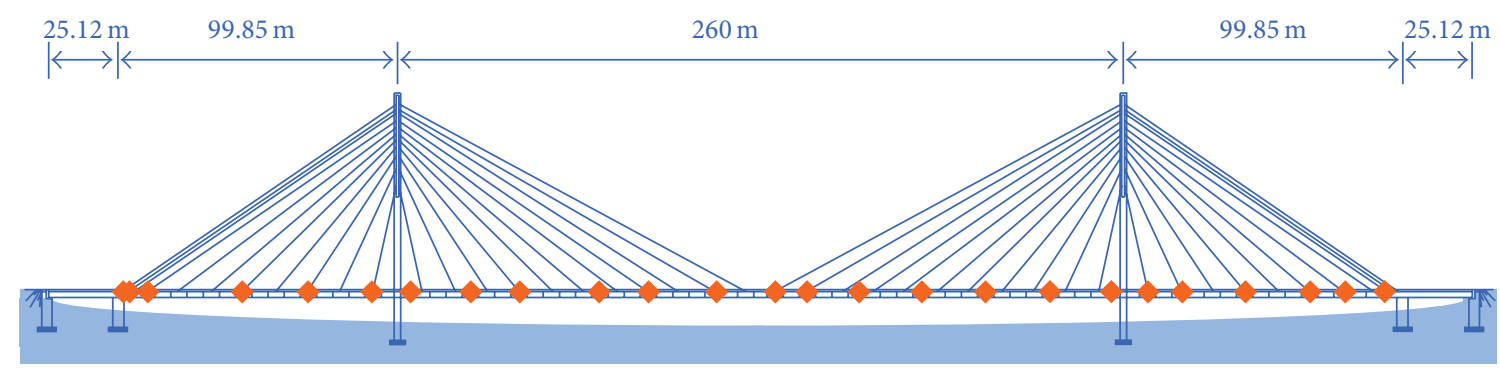

FIgURE 9: The optimal sensor configuration extracted by GA with the MAC.

It should be noted that all the analyses in this paper are conducted based on the assumed theoretical models while the real environment is more complex. The advanced SHM requires a more versatile sensor configuration to comprehensively understand the performance of a structure; therefore, developing algorithms for multiobjective optimization may be a good direction for future work.

\section{Conflict of Interests}

The authors declare that there is no conflict of interests regarding the publication of this paper.

\section{Acknowledgments}

This research work was jointly supported by the 973 Program (Grant no. 2015CB060000), the National Natural Science Foundation of China (Grants nos. 51421064, 51478081, and 51222806), the Fok Ying Tong Education Foundation (Grant no. 141072), and the Science Fund for Distinguished Young Scholars of Dalian (Grant no. 2014J11JH125).

\section{References}

[1] L. Faravelli, D. Bortoluzzi, T. B. Messervey, and L. Sasek, "Temperature effects on the response of the bridge "ÖBB Brücke Großhaslau'”' in Mechanics and Model-Based Control of Advanced Engineering Systems, pp. 85-94, Springer, Vienna, Austria, 2014.

[2] Y. Fujino, "Vibration, control and monitoring of long-span bridges-recent research, developments and practice in Japan," Journal of Constructional Steel Research, vol. 58, no. 1, pp. 71-97, 2002.
[3] B. Chen, S. L. Zhao, and P. Y. Li, "Application of HilbertHuang transform in structural health monitoring: a state-ofthe-art review," Mathematical Problems in Engineering, vol. 2014, Article ID 317954, 22 pages, 2014.

[4] Y. L. Xu, B. Chen, C. L. Ng, K. Y. Wong, and W. Y. Chan, "Monitoring temperature effect on a long suspension bridge," Structural Control and Health Monitoring, vol. 17, no. 6, pp. 632653, 2010.

[5] G.-D. Zhou and T.-H. Yi, "Recent developments on wireless sensor networks technology for bridge health monitoring," Mathematical Problems in Engineering, vol. 2013, Article ID 947867, 33 pages, 2013.

[6] J. M. Ko and Y. Q. Ni, "Technology developments in structural health monitoring of large-scale bridges," Engineering Structures, vol. 27, no. 12, pp. 1715-1725, 2005.

[7] G.-D. Zhou and T.-H. Yi, "A summary review of correlations between temperatures and vibration properties of long-span bridges," Mathematical Problems in Engineering, vol. 2014, Article ID 638209, 19 pages, 2014.

[8] T.-H. Yi, H.-N. Li, and X.-D. Zhang, "Health monitoring sensor placement optimization for canton tower using immune monkey algorithm," Structural Control and Health Monitoring, 2014.

[9] D. S. Li, G. X. Wang, H. N. Li, L. Ren, and G. B. Song, "On optimal sensor placement for structural health monitoring by subspace approximation," in Proceedings of the 11th Aerospace Division International Conference on Engineering, Science, Construction, and Operations in Challenging Environments, 2008.

[10] M. Meo and G. Zumpano, "On the optimal sensor placement techniques for a bridge structure," Engineering Structures, vol. 27, no. 10, pp. 1488-1497, 2005.

[11] C. Papadimitriou, Y. Haralampidis, and K. Sobczyk, "Optimal experimental design in stochastic structural dynamics," Probabilistic Engineering Mechanics, vol. 20, no. 1, pp. 67-78, 2005.

[12] T.-H. Yi and H.-N. Li, "Methodology developments in sensor placement for health monitoring of civil infrastructures," 
International Journal of Distributed Sensor Networks, vol. 2012, Article ID 612726, 11 pages, 2012.

[13] L. Yao, W. A. Sethares, and D. C. Kammer, "Sensor placement for on-orbit modal identification via a genetic algorithm," AIAA Journal, vol. 31, no. 10, pp. 1922-1928, 1993.

[14] K. Worden and A. P. Burrows, "Optimal sensor placement for fault detection," Engineering Structures, vol. 23, no. 8, pp. 885901, 2001.

[15] S.-F. Hwang and R.-S. He, "A hybrid real-parameter genetic algorithm for function optimization," Advanced Engineering Informatics, vol. 20, no. 1, pp. 7-21, 2006.

[16] T. G. Carne and C. R. Dohrmann, "A modal test design strategy for modal correlation," in Proceedings of the 13th International Modal Analysis Conference, vol. 317, pp. 927-933, Schenectady, NY, USA, February 1995.

[17] M. Huang, J. Li, and H. Zhu, "Optimal sensor layout for bridge health monitoring based on dual-structure coding genetic algorithm," in Proceedings of the International Conference on Computational Intelligence and Software Engineering (CiSE '09), pp. 1-4, Wuhan, China, December 2009.

[18] T.-H. Yi, H.-N. Li, and M. Gu, "Optimal sensor placement for health monitoring of high-rise structure based on genetic algorithm," Mathematical Problems in Engineering, vol. 2011, Article ID 395101, 11 pages, 2011.

[19] T.-H. Yi, H.-N. Li, and M. Gu, "Optimal sensor placement for structural health monitoring based on multiple optimization strategies," Structural Design of Tall and Special Buildings, vol. 20, no. 7, pp. 881-900, 2011.

[20] G.-D. Zhou and T.-H. Yi, "The node arrangement methodology of wireless sensor networks for long-span bridge health monitoring," International Journal of Distributed Sensor Networks, vol. 2013, Article ID 865324, 8 pages, 2013.

[21] G.-D. Zhou and T.-H. Yi, “The nonuniform node configuration of wireless sensor networks for long-span bridge health monitoring," International Journal of Distributed Sensor Networks, vol. 2013, Article ID 797650, 9 pages, 2013.

[22] G. D. Zhou, T. H. Yi, and H. N. Li, "Wireless sensor placement for bridge health monitoring using a generalized genetic algorithm," International Journal of Structural Stability and Dynamics, vol. 14, no. 5, Article ID 1440011, 21 pages, 2014.

[23] P. N. Ngatchou, W. L. J. Fox, and M. A. El-Sharkawi, "Distributed sensor placement with sequential particle swarm optimization," in Proceedings of the IEEE Swarm Intelligence Symposium (SIS '05), pp. 395-398, June 2005.

[24] A. R. M. Rao and G. Anandakumar, "Optimal placement of sensors for structural system identification and health monitoring using a hybrid swarm intelligence technique," Smart Materials and Structures, vol. 16, no. 6, pp. 2658-2672, 2007.

[25] T.-H. Yi, H.-N. Li, and X.-D. Zhang, "A modified monkey algorithm for optimal sensor placement in structural health monitoring," Smart Materials and Structures, vol. 21, no. 10, Article ID 105033, 9 pages, 2012.

[26] T. H. Yi, H. N. Li, and X. D. Zhang, "Sensor placement on Canton tower for health monitoring using asynchronousclimbing monkey algorithm," Smart Materials and Structures, vol. 21, no. 12, Article ID 125023, pp. 1-12, 2012.

[27] X. S. Yang, Nature-Inspired Metaheuristic Algorithm, Luniver Press, Beckington, UK, 2008.

[28] X.-S. Yang, "Firefly algorithms for multimodal optimization," in Stochastic Algorithms: Foundations and Applications, vol. 5792 of Lecture Notes in Computer Science, pp. 169-178, Springer, Berlin, Germany, 2009.
[29] A. H. Gandomi, X.-S. Yang, and A. H. Alavi, "Mixed variable structural optimization using firefly algorithm," Computers \& Structures, vol. 89, no. 23-24, pp. 2325-2336, 2011.

[30] I. Fister, I. Fister Jr., X.-S. Yang, and J. Brest, "A comprehensive review of firefly algorithms," Swarm and Evolutionary Computation, vol. 13, pp. 34-46, 2013.

[31] R. W. Hamming, "Error detecting and error correcting codes," The Bell System Technical Journal, vol. 29, pp. 147-160, 1950.

[32] J. H. Holland, Adaption in Natural and Artificial Systems, University of Michigan Press, Ann Arbor, Mich, USA, 1975.

[33] K. P. Ferentinos and T. A. Tsiligiridis, "Adaptive design optimization of wireless sensor networks using genetic algorithms," Computer Networks, vol. 51, no. 4, pp. 1031-1051, 2007.

[34] W. Liu, Z. Hou, and M. A. Demetriou, "A computational scheme for the optimal sensor/actuator placement of flexible structures using spatial $\mathrm{H}_{2}$ measures," Mechanical Systems and Signal Processing, vol. 20, no. 4, pp. 881-895, 2006.

[35] http://smc.hit.edu.cn/index.php?option=com_content\&view= article\&id $=156 \&$ Itemid $=81$.

[36] H. Li, C. M. Lan, Y. Ju, and D. S. Li, "Experimental and numerical study of the fatigue properties of corroded parallel wire cables," Journal of Bridge Engineering, vol. 17, no. 2, pp. 211220, 2012. 

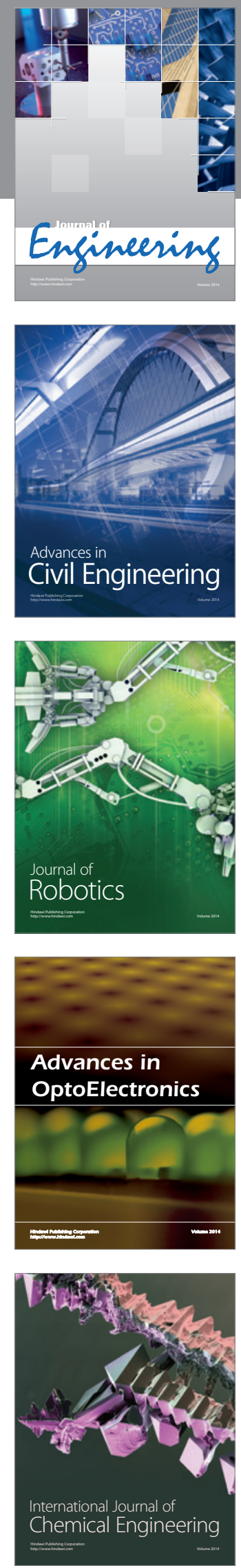

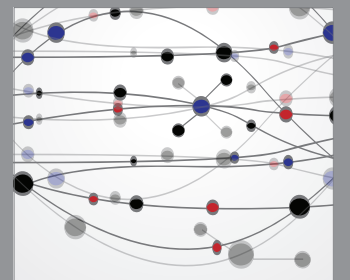

The Scientific World Journal
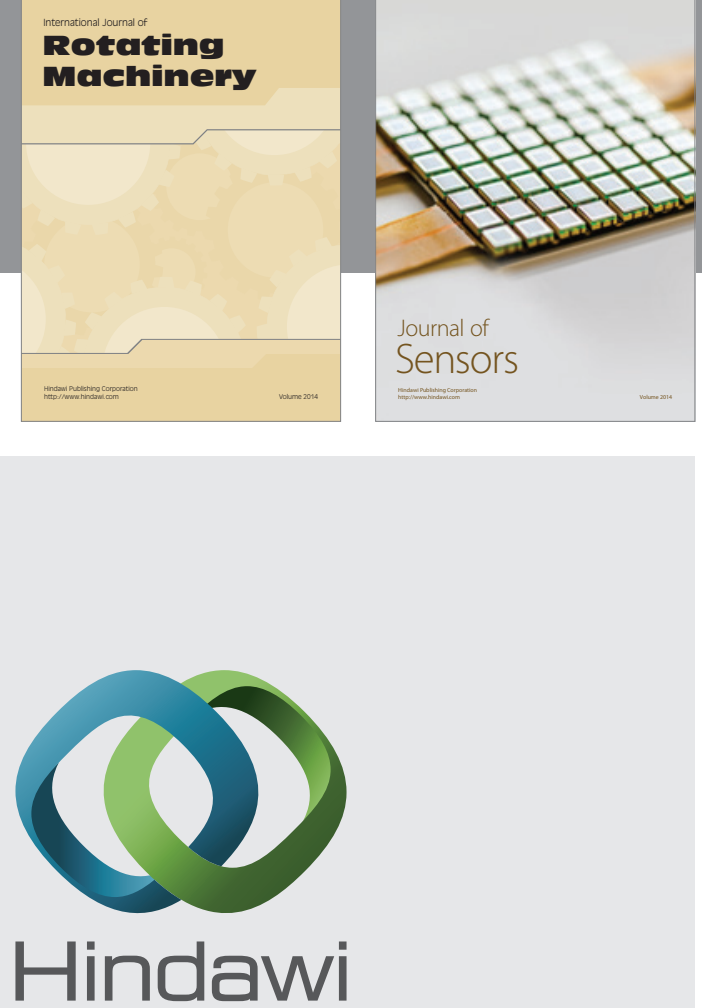

Submit your manuscripts at http://www.hindawi.com
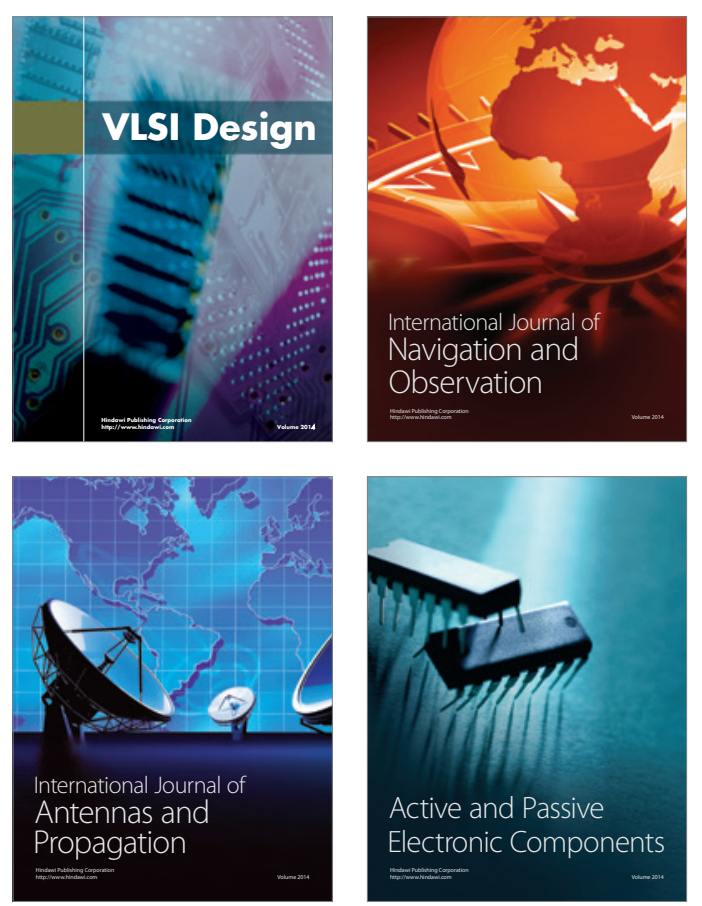
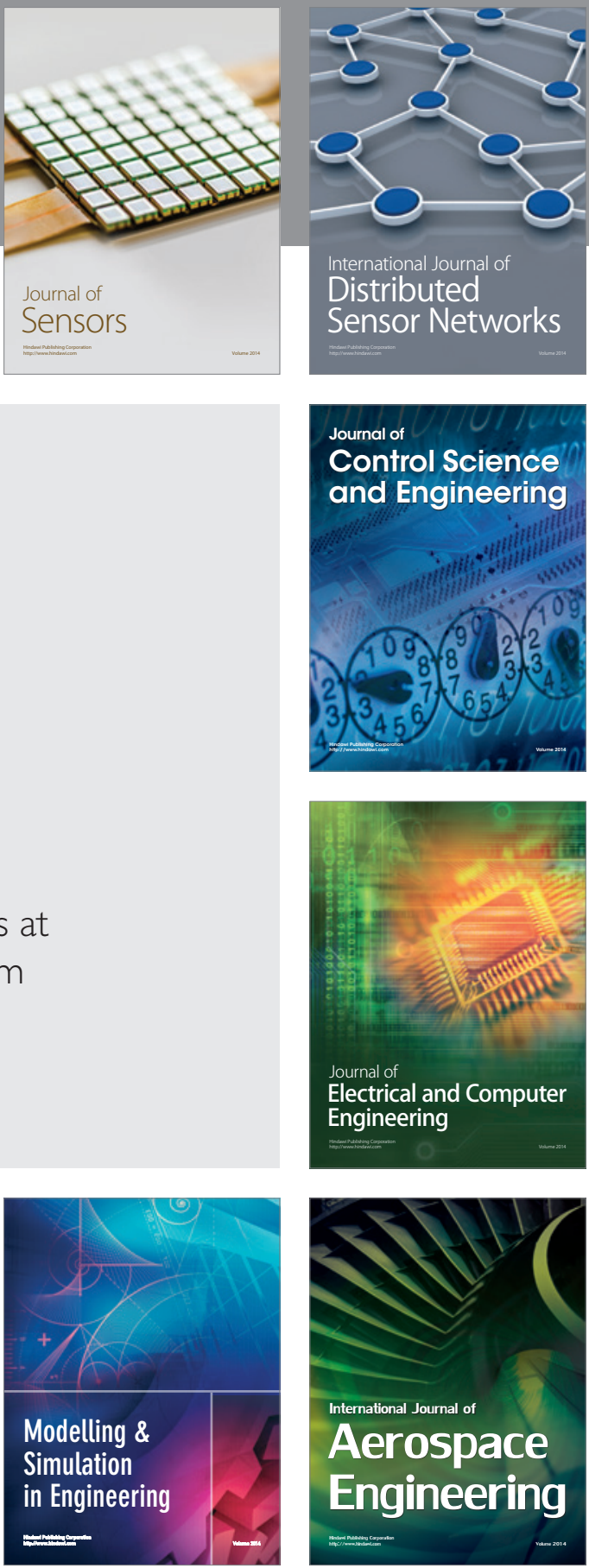

Journal of

Control Science

and Engineering
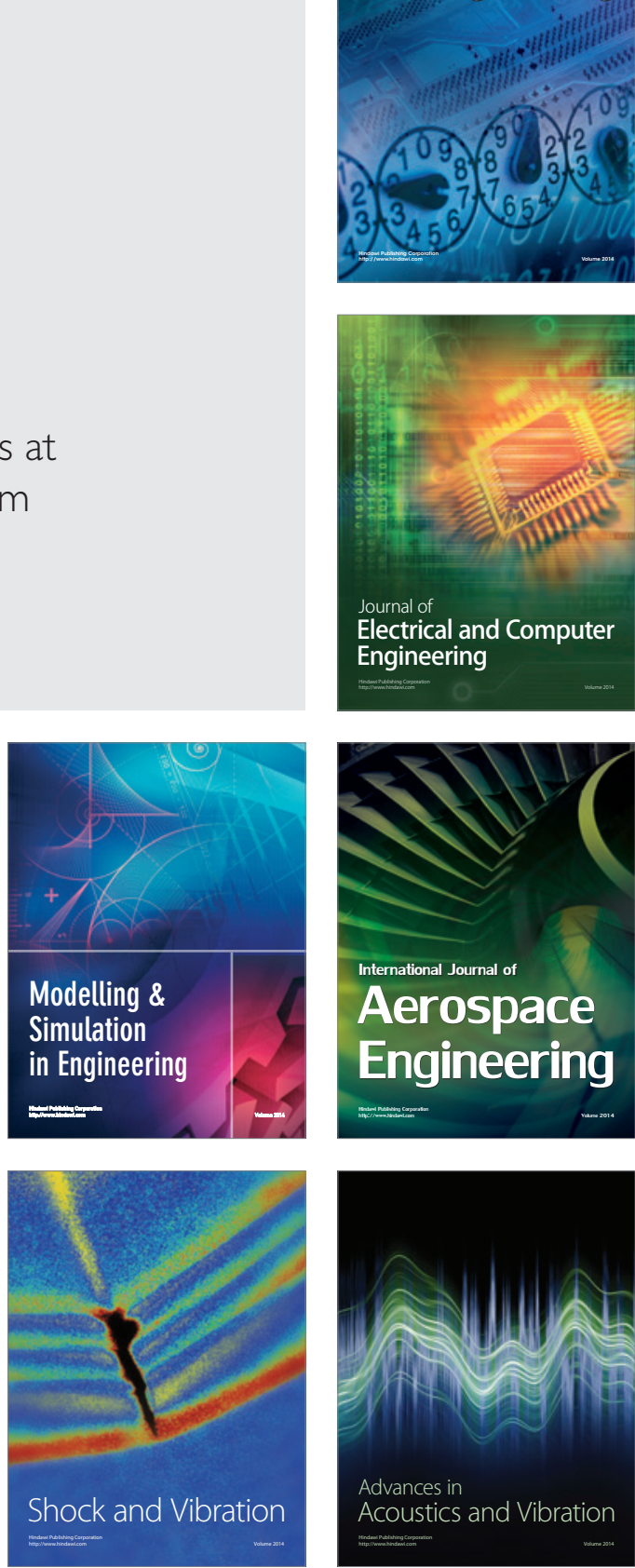